\title{
Escala diagramática para avaliação da severidade da antracnose em pinha
}

\author{
Diagrammatic scale for anthracnose assessment severity in custard apple
}

\author{
Kamila Câmara Correia ${ }^{I}$ Ricardo Brainer Martinss ${ }^{I I}$ Marcos Paz Saraiva Câmara \\ Sami Jorge Michereff ${ }^{1^{*}}$
}

\begin{abstract}
A antracnose, causada por Colletotrichum gloeosporioides, é uma importante doença da pinha (Annona squamosa) no Brasil e em nível mundial. Devido à inexistência de métodos padronizados para quantificação dessa doença, foi desenvolvida uma escala diagramática com níveis de 1, 2, 5, 10, 20 e 40\% de área foliar lesionada. A escala diagramática foi validada por 10 avaliadores, baseado em 48 folhas de pinha com diferentes niveis de severidade da antracnose, mensurados previamente com o programa Assess ${ }^{\circledR}$. A acurácia, a precisão e a reprodutibilidade das estimativas de cada avaliador foi determinada por regressão linear simples entre a severidade real e a estimada, com e sem o auxílio da escala. Com a escala, os avaliadores obtiveram melhores niveis de acurácia e precisão das estimativas, com os erros absolutos concentrando-se na faixa de $10 \%$. Os avaliadores apresentaram elevada repetibilidade $(91 \%)$ e reprodutibilidade $(\geq 90 \% \mathrm{em}$ $94,5 \%$ dos casos) das estimativas com a utilização da escala. Portanto, a escala diagramática proposta é adequada para avaliação da severidade da antranose da pinha.
\end{abstract}

Palavras-chave: Annona squamosa, Colletotrichum gloeosporioides, fitopatometria.

\section{ABSTRACT}

Anthracnose caused by Colletotrichum gloeosporioides is an important disease of custard apple (Annona squamosa) in Brazil and worldwide. Due to the inexistence of standard methods for the assessment of that disease, a diagrammatic scale was developed with 1, 2, 5, 10, 20 and $40 \%$ of diseased leaf area. The diagrammatic scale was validated by 10 raters using 48 custard apple leaves with different levels of anthracnose severity previously measured by the software Assess ${ }^{\circledR}$. The accuracy, precision and reproducibility estimative of each rater were determined by simple linear regression between actual and estimated severity, with and without the use of the scale. With the scale, the raters obtained better levels of accuracy and precision, with absolute errors concentrating around 10\%. Raters showed high repeatability (91\%) and reproducibility ( $\geq 90 \%$ in $94.5 \%$ of the cases) of the estimates by using the scale. The proposed diagrammatic scale is suitable for the evaluation of anthracnose severity in custard apple.

Key words: Annona squamosa, Colletotrichum gloeosporioides, phytopatometry.

A pinha (Annona squamosa L.) é uma anonácea de importância econômica em diversos países. No Brasil, em 2006 foram cultivados cerca de 5.000ha e produzidas 14.000ton de frutas, destacandose como maiores produtores os estados da Bahia e São Paulo (IBGE, 2010). A antracnose, causada por Colletotrichum gloeosporioides (Penz.) Sacc., é a doença mais comum e destrutiva da pinha no Brasil (CARDOSO \& FREIRE, 2003; JUNQUEIRA et al., 2001) e em nível mundial (PLOETZ, 2003). A doença pode provocar até $70 \%$ de perdas de frutos quando as chuvas são prolongadas durante a floração e a formação de frutos (JUNQUEIRA et al., 2001). Os sintomas ocorrem nas folhas, flores e frutos. Nas folhas, ocorrem inicialmente manchas pardas, com aspecto aquoso, que assumem coloração escura, provocando a distorção e a queima do limbo foliar, podendo levar à queda

IDepartamento de Agronomia, Área de Fitossanidade, Universidade Federal Rural de Pernambuco (UFRPE), Av. Dom Manoel de Medeiros, s/n, 52171-900, Recife, PE, Brasil. E-mail: sami@depa.ufrpe.br. *Autor para correspondência.

"Campus de Arapiraca, Universidade Federal de Alagoas (UFAL), Arapiraca, AL, Brasil. 
prematura das folhas. A infecção pode se estender às flores, necrosando as partes florais ou mumificando os frutos recém formados, que caem ao solo (CARDOSO \& FREIRE, 2003; PLOETZ, 2003).

Apesar da importância da antracnose em pinha, inexistem métodos padronizados para quantificação dessa doença, o que limita a realização de estudos epidemiológicos e de comparação de medidas de controle. Nesse contexto, a utilização de uma escala diagramática pode constituir ferramenta eficiente para padronização das avaliações da severidade da doença. Escalas diagramáticas, quando cuidadosamente desenvolvidas e validadas, propiciam estimativas acuradas, precisas e reprodutíveis dos níveis de severidade das doenças. A acurácia refere-se à proximidade de uma estimativa a um valor real de quantidade de doença avaliada, a precisão refere-se à variação ou repetibilidade associadas com uma estimativa, e a reprodutibilidade refere-se à ausência de variação em estimativas quando a mesma amostra de doença é avaliada por outro avaliador (NUTTER JR., 2002).

Diante do exposto, este trabalho teve como objetivos desenvolver uma escala diagramática para avaliação da severidade da antracnose em folhas de pinha e analisar os níveis de acurácia, precisão e reprodutibilidade das estimativas geradas com sua utilização.

Para elaboração da escala diagramática foram coletadas 100 folhas de pinha (cv. Pinha AP) em plantio no município de Recife - PE, com diferentes níveis de severidade da antracnose. As folhas foram digitalizadas a 200 dpi e, com auxílio do programa Assess ${ }^{\circledR}$ (The American Phytopathological Society, St. Paul, MN, USA), foi determinada a porcentagem de área lesionada de cada folha, representando o grau de severidade da doença. Uma escala diagramática foi confeccionada baseada em quatro fatores presentes nas 100 folhas coletadas: valor máximo de severidade da doença, forma, distribuição e freqüência das lesões. A escala confeccionada possui seis níveis de severidade.

$\mathrm{Na}$ validação da escala diagramática foram utilizadas 48 folhas de pinha com diferentes níveis de severidade da antracnose, reproduzidas em fotocópias coloridas. A severidade foi avaliada por 10 pessoas (A a J) sem experiência na quantificação da doença. Inicialmente, a severidade foi estimada sem o auxílio da escala diagramática e, após sete dias, foi realizada uma primeira avaliação com o auxílio da escala. Visando avaliar a repetibilidade das estimativas com a escala diagramática, sete dias após a primeira avaliação, nova seqüência dos mesmas folhas foi organizada e uma segunda estimativa visual com auxílio da escala foi efetuada pelos mesmos avaliadores.
A acurácia, a precisão e a reprodutibilidade das estimativas foram determinadas por regressão linear simples, considerando a severidade real como variável independente e a severidade estimada como variável dependente. A acurácia das estimativas de cada avaliador e do conjunto de avaliadores foi determinada pelo teste $t$ aplicado ao intercepto da regressão linear (a), para verificar a hipótese Ho: $a=0$, e ao coeficiente angular da reta $(b)$, para testar a hipótese Ho: $b=1$, ao nível $5 \%$ de probabilidade $(\mathrm{P}=0,05)$. A precisão das estimativas foi determinada pelo coeficiente de determinação da regressão $\left(R^{2}\right)$, pela variância dos erros absolutos (severidade estimada menos severidade real) e pela repetibilidade das estimativas, determinada pela regressão da segunda avaliação em relação à primeira de uma mesma unidade amostral. A reprodutibilidade das estimativas foi determinada pelos valores de $R^{2}$ obtidos de regressões lineares entre as severidades estimadas da mesma unidade amostral por diferentes avaliadores combinados em pares (NUTTER JR. \& SCHULTZ, 1995).

O valor máximo de severidade da antracnose verificado nas 100 folhas de pinha foi de 39,9\%, enquanto o valor mínimo foi de $0,28 \%$. Considerando esses limites, a escala diagramática elaborada para quantificação da severidade da doença foi representada pelos níveis de 1, 2, 5, 10, 20 e 40\% de área foliar lesionada (Figura 1). Na primeira etapa de validação da escala diagramática, ou seja, sem a sua utilização, os valores do intercepto de todos os avaliadores diferiram significativamente de zero para as retas de regressão entre a severidade real e a estimada, com valor médio de 5,94 (Tabela 1). Com a utilização da escala, os valores do intercepto de somente $30 \%$ dos avaliadores na primeira avaliação e $20 \%$ na segunda avaliação se mantiveram significativamente diferentes de zero, mas com valores inferiores aos constatados sem a utilização da escala. Em todas essas situações, os avaliadores superestimaram a severidade da antracnose. Os valores do coeficiente angular da reta de $80 \%$ dos avaliadores diferiram significativamente de 1 sem a utilização da escala diagramática, enquanto com a utilização da escala houve redução para $20 \%$ na primeira avaliação e $0 \%$ na segunda avaliação (Tabela 1 ), indicando elevados níveis de acurácia das estimativas com a utilização da escala diagramática.

A análise da precisão das estimativas visuais da severidade sem a utilização da escala diagramática justificou de 52 a $73 \%$ da variação $\left(R^{2}\right)$ na mensuração eletrônica da doença, com média de $66 \%$ (Tabela 1 ). Com a utilização da escala, na primeira avaliação, as estimativas visuais explicaram 89 a 93\% da variação na mensuração eletrônica, com média de $91 \%$, enquanto 


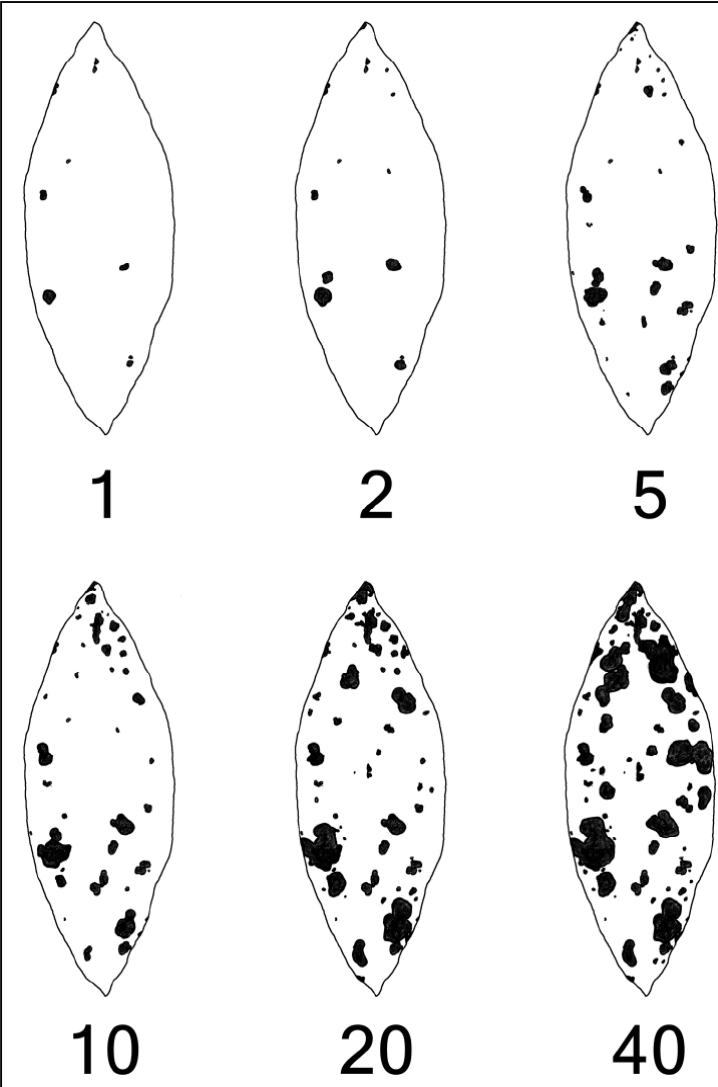

Figura 1 - Escala diagramática para avaliação de severidade da antracnose em pinha, indicando os níveis de $1,2,5,10,20$ e $40 \%$ de área lesionada. na segunda avaliação as estimativas visuais explicaram 91 a $96 \%$ da variação, com média de $93 \%$. A maioria dos avaliadores obteve excelente repetibilidade nas estimativas com a escala diagramática, pois a quantidade média de variação na primeira avaliação explicada pela segunda avaliação foi de $91 \%$. A melhoria da precisão das estimativas na segunda avaliação com a escala em relação à primeira indica que, além da utilização da escala, a familiarização dos avaliadores com a doença pode melhorar a acurácia e a precisão das estimativas (NUTTER JR., 2002).

Houve redução nos erros absolutos para as estimativas com o auxílio da escala diagramática, quando comparada com a distribuição dos resíduos das estimativas obtidas sem a escala. Nas duas avaliações com o auxílio da escala, os resíduos concentraram-se na faixa de $10 \%$, considerados aceitáveis em estudos de avaliação de escalas diagramáticas e que podem ser reduzidos com o treinamento dos avaliadores (NUTTER JR. \& SCHULTZ, 1995). Por outro lado, sem a utilização da escala, os erros concentraram-se na faixa de $25 \%$, sendo considerados elevados. Sem a utilização da escala diagramática, a reprodutibilidade das estimativas da severidade da doença foi $\geq 90 \%$ em somente $8,5 \%$ dos casos, enquanto com o uso da escala diagramática esse nível foi atingido em $74,5 \%$ dos casos na primeira avaliação e $94,5 \%$ dos casos na segunda.

O único estudo de validação de escala diagramática para quantificação da severidade da

Tabela 1 - Estimativa do intercepto $(a)$, do coeficiente angular da reta $(b)$ e do coeficiente de determinação $\left(R^{2}\right)$ de equações de regressão linear simples relacionando estimativas visuais da antracnose em pinha efetuadas por dez avaliadores, sem e com o auxílio da escala diagramática, à severidade real determinada eletronicamente com auxílio do programa Assess ${ }^{\circledR}$.

\begin{tabular}{|c|c|c|c|c|c|c|c|c|c|}
\hline \multirow{3}{*}{ Avaliador } & \multirow{2}{*}{\multicolumn{3}{|c|}{ 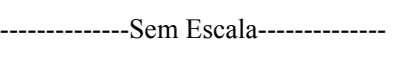 }} & \multirow{2}{*}{\multicolumn{3}{|c|}{ - }} & \multirow{2}{*}{\multicolumn{3}{|c|}{----------2 }} \\
\hline & & & & & & & & & \\
\hline & $\mathrm{a}$ & $\mathrm{b}$ & $\mathrm{R}^{2}$ & $\mathrm{a}$ & $\mathrm{b}$ & $\mathrm{R}^{2}$ & $\mathrm{a}$ & $\mathrm{b}$ & $\mathrm{R}^{2}$ \\
\hline A & $4,99 *$ & $0,74^{*}$ & 0,65 & 1,53 & 0,86 & 0,92 & 0,71 & 0,99 & 0,93 \\
\hline B & $6,71^{*}$ & $0,81^{*}$ & 0,68 & $3,20^{*}$ & $0,79 *$ & 0,89 & $2,22 *$ & 0,88 & 0,91 \\
\hline $\mathrm{C}$ & $8,32 *$ & $1,38^{*}$ & 0,65 & 1,23 & 0,90 & 0,92 & 0,59 & 1,14 & 0,96 \\
\hline $\mathrm{D}$ & $2,64^{*}$ & $0,73 *$ & 0,71 & $2,52 *$ & 0,89 & 0,91 & 0,87 & 0,98 & 0,94 \\
\hline $\mathrm{E}$ & $12,07^{*}$ & $0,60 *$ & 0,52 & 1,45 & 0,91 & 0,91 & 1,48 & 0,94 & 0,91 \\
\hline $\mathrm{F}$ & $3,16^{*}$ & $0,78^{*}$ & 0,65 & $-0,31$ & 0,87 & 0,92 & 0,73 & 0,99 & 0,93 \\
\hline G & $4,81^{*}$ & $1,47^{*}$ & 0,73 & 0,20 & 1,01 & 0,90 & $-0,51$ & 0,90 & 0,91 \\
\hline $\mathrm{H}$ & $2,45^{*}$ & 0,88 & 0,68 & $-0,44$ & 1,14 & 0,93 & $-0,07$ & 1,05 & 0,95 \\
\hline I & $3,71^{*}$ & $0,83^{*}$ & 0,70 & 1,32 & 0,90 & 0,91 & 0,68 & 1,01 & 0,92 \\
\hline $\mathrm{J}$ & $10,57^{*}$ & 0,86 & 0,59 & $3,34 *$ & $0,80 *$ & 0,90 & $1,84 *$ & 0,93 & 0,91 \\
\hline Média & 5,94 & 0,91 & 0,66 & 1,40 & 0,91 & 0,91 & 0,85 & 0,98 & 0,93 \\
\hline
\end{tabular}

*Asterisco representa situações em que a hipótese de nulidade $(\mathrm{a}=0 \mathrm{ou} \mathrm{b}=1)$ foi rejeitada pelo teste $t(\mathrm{P}=0,05)$.

Ciência Rural, v.41, n.1, jan, 2011. 
antracnose em anonáceas foi realizado no México, utilizando folhas de graviola (Annona cherimola Mill.) (TOVAR-SOTO et al., 2002). Nesse estudo não foram efetuadas avaliações sem a escala, mas quando essa foi utilizada pela primeira vez, os níveis de precisão $(R=0,20-0,70)$ e acurácia $(b=0,40-1,30)$ foram inadequados. Quando quatro avaliadores que participaram da primeira avaliação utilizaram a escala novamente, três avaliadores mantiveram desempenhos muitos ruins de precisão $\left(R^{2}=0,59-0,75\right)$ e acurácia ( $b=0,69-0,80)$, indicando que a escala não propiciou melhorias nas estimativas da severidade da doença. Portanto, apesar de estar disponível para utilização, essa escala de antracnose da graviola não deve ser utilizada para quantificação da antracnose em pinha, tendo em vista os baixos níveis de acurácia e precisão das estimativas propiciados com sua utilização. Por outro lado, a escala diagramática proposta no presente estudo para quantificação da severidade da antracnose em folhas de pinha propiciou melhorias significativas nos níveis de acurácia, precisão e reprodutibilidade das estimativas, podendo ser utilizada em estudos epidemiológicos e de comparação de medidas de controle da doença.

\section{REFERÊNCIAS}

CARDOSO, J.E.; FREIRE, F.C.O. Doenças das anonáceas. In: FREIRE, F.C.O. et al. (Ed.). Doenças de fruteiras tropicais de interesse agroindustrial. Brasília: Embrapa Informação Tecnológica, 2003. p.145-189.

IBGE (Instituto Brasileiro de Geografia e Estatística). Sidra: sistema IBGE de recuperação automática - banco de dados agregados, 2010. Disponível em: http://www.sidra.ibge.gov.br/ bda/agric/default.asp ? t $=2 \& z=t \& o=11 \& u 1=1 \&$ $\mathrm{u} 2=1 \& u 3=1 \& u 4=1 \& u 5=1 \& u 6=1$. Acesso em: 02 set. 2010.

JUNQUEIRA, N.T.V. et al. Principais doenças da fruteirado-conde no cerrado. Planaltina: Embrapa Cerrados, 2001. 33p. (Circular Técnica, 16).

NUTTER JR., F.W. Disease assessment. In: MALOY, O.C.; MURRAY, T.D. (Ed.). Encyclopedia of plant pathology. New York: Wiley, 2002. p.312-323.

NUTTER JR., F.W.; SCHULTZ, P.M. Improving the accuracy and precision of disease assessments: selection of methods and use of computer-aided training programs. Canadian Journal of Plant Pathology, v.17, n.1, p.174-184, 1995.

PLOETZ, R.C. Diseases of atemoya, cherimoya, soursop, sugar apple and related fruit crops. In: PLOETZ, R.C. (Ed.). Diseases of tropical fruit crops. Wallingford: CABI, 2003. p.21-34.

TOVAR-SOTO, A. et al. Escala logarítimica diagramática de severidad de la mancha negra (Colletotrichum gloeosporioides Penz.) en chirimoyo (Annona cherimola Mill). Revista Mexicana de Fitopatología, v.20, n.1, p.103-109, 2002. Disponível em: $<\mathrm{http}: / / \mathrm{www}$.sociedadmexicanadefitopatologia.org/ archives/61220117.pdf $>$. Acesso em: 11 dez. 2008. 